\section{La inflamación en la hipertensión arterial, mecanismos de producción e implicancias terapéuticas}

\author{
HERNÁN PRAT ${ }^{1 *}$, PATRICIO ARAOS ${ }^{2, a^{*}}$, LUIS MICHEA ${ }^{3,4,5}$
}

\section{Role of inflammation in hypertension}

Even though the mechanisms that mediate essential hypertension (HT) are not fully understood, an immunological-inflammatory mechanism could be the common pathway for diverse pathophysiological mechanisms. We analyze in a simplified way the participation of the immune system in HT. T lymphocytes (TL) and antigen presenting cells (APCs) are components of the immune system capable of generating proinflammatory cytokines. They cause endothelial damage, vasoconstriction, and decreased urinary sodium excretion. CD4+ and CD8+ TL are effector cells, causally implicated in the development of $H T$, whereas type $\gamma \delta$ TL play their pathogenic role in HT enhancing endothelial dysfunction. Additionally, a immunomodulation decrease by regulatory $T L$, worsens endothelial dysfunction and reduces vasodilation in experimental HT. Results of recent studies indicate that lymphocyte activation would be mediated by antigens captured by antigen APCs for subsequent presentation to "naive" TL. On the other hand, proinflammatory states such as obesity, the change of the intestinal microbiota and the increase in salt intake favors TL and APC activation, contributing to $H T$ development.

(Rev Med Chile 2021; 149: 255-262)

Key words: Cytokines; Lymphocytes; Hypertension; Inflammation.

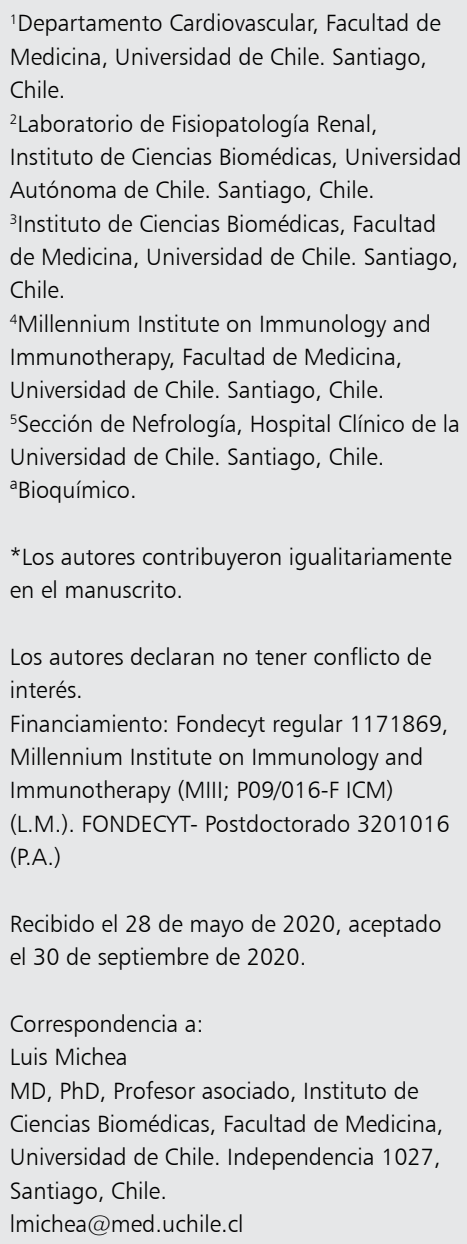

$\mathrm{D}$ esde el siglo pasado se propusieron mecanismos para producción de la HTA, mostrando al riñón como causante, debido a un defecto en la excreción renal de sodio. Posteriormente, se descubrió la importancia del sistema renina-angiotensina-aldosterona (SRAA) en la regulación de la presión arterial (PA) y del equilibrio hidroelectrolítico. Últimamente junto al estrés oxidativo, aparecen nuevos mecanismos que implican células inmunológicas y sus mediadores en el desarrollo de HTA.

\section{Sistema inmune como agente causal de HTA}

La HTA es un estado subinflamatorio crónico, con aumento de factores proinflamatorios cir- culantes e infiltración de células mononucleares (linfocitos, células del sistema monocito-macrofágico) en tejidos diana de daño hipertensivo. Además de mediar la inflamación-fibrosis tisular, las células de inmunidad innata y adaptativa tendrían un rol causal en la HTA ${ }^{1}$. Considerando que la HTA depende del aumento del gasto cardíaco (GC) y/o de la resistencia periférica total (RPT), el objetivo de esta revisión narrativa es analizar la modulación de la función vascular y renal por componentes del sistema inmune y cómo ésta se interrelaciona con medidas terapéuticas.

\section{Inmunidad adaptativa}

Maniobras y/o fármacos inmunosupresores que disminuyen el recuento y actividad linfocitaria 
protegen del desarrollo de HTA experimental, aminorando la infiltración celular mononuclear, el daño tisular, el desarrollo de hipertrofia y fibrosis cardiaca $^{2}$. La ausencia genética de linfocitos protege parcialmente de HTA en animales sal-sensibles, en respuesta a Angiotensina II (AngII) o mineralocorticoides+dieta rica en sal $(\mathrm{MNa})^{3}$.

\section{a) Papel de los linfocitos en la alteración de la función vascular en HTA}

La HTA experimental, inducida por AngII, $\mathrm{MNa}$ o ingesta de sal, causa estrés oxidativo en la pared arterial, disfunción endotelial, fibrosis e hipertrofia de la túnica media. Todas estas alteraciones contribuyen al aumento de la RPT y son prevenidas por la eliminación genética de $\mathrm{LT}^{1-3}$.

Diversas familias linfocitarias participan en el daño vascular hipertensivo. La activación de $\mathrm{LTCD}^{+}$, que son citotóxicos y median respuesta inmune al activarse con el complejo mayor de histocompatibilidad I (MHCI), contribuye al aumento de RPT en HTA experimental. La eliminación genética de ellos protege del desarrollo de HTA experimental; la transferencia adoptiva de $\mathrm{LTCD}^{+}$provenientes de un animal hipertenso a un animal sano causa aumentos de la PA en el animal receptor ${ }^{4}$. Este tipo de linfocitos es necesario para que se desarrolle estrés oxidativo, disfunción endotelial y remodelación de la pared arterial. También son inductores de inflamación y fibrosis en el miocardio de animales hipertenos ${ }^{5}$. Adicionalmente, la deleción del receptor de mineralocorticoide (MR) de los LTCD8 ${ }^{+}$disminuye la producción de interferón-gamma (INF- $\gamma$ ), preservándose la función endotelial y aminorando el aumento de PA al infundir AngII ${ }^{6}$.

Linfocitos $\mathrm{T} \mathrm{CD}^{+}$del tipo helper proinflamatorios LTh1 y LTh17, que coordinan la respuesta inmune adaptativa, también contribuyen al aumento de la RPT a través de la secreción de diversas citoquinas ${ }^{1-3}$. Estudios experimentales han demostrado que la expresión de citoquinas características de LTh1 y LTh17 como interferon gama (INF- $\gamma$ ), Factor de Necrosis Tumoral $\alpha$ $(\mathrm{TNF} \alpha)$, Interleuquina 6 (IL-6) e Interleuquina 17A (IL17) es necesaria para que se produzca disfunción endotelial, infiltración de la adventicia vascular con células mononucleares, fibrosis y e hipertrofia de la túnica media arterial en HTA experimental ${ }^{7,8}$. La deficiencia de IL-6 o de INF- $\gamma$ protege parcialmente de la disfunción endotelial, reduce la infiltración de LT y macrófagos, la hipertrofia y fibrosis cardiaca en el corazón de ratones tratados con AngII ${ }^{8,9}$. Además, el bloqueo de los receptores para estas citoquinas protege del aumento de PA por AngII, mineralocorticoides o dieta rica en sal ${ }^{10,11}$.

Junto con el aumento de actividad de LTh1 y LTh17, en la HTA experimental se produce disminución de la actividad de LT reguladores (LTreg), que tienen acción inmunomoduladora y anti-inflamatoria ${ }^{7}$. La transferencia adoptiva de LTreg a ratones hipertensos por AngII o Aldosterona previene el desarrollo de disfunción endotelial, la fibrosis e hipertrofia de la pared arterial ${ }^{12}$. La acción protectora de los LTreg se ha atribuido a la secreción de IL-10 inmunomoduladora, hipótesis refutada por estudios recientes que muestran que la deficiencia de IL-10 protege del desarrollo de HTA experimental ${ }^{13}$.

La producción de IgG por Linfocitos B (LB) también tendría acciones deletéreas sobre la pared arterial, que contribuyen al aumento de PA. El desarrollo de HTA se asocia con aumento de los niveles plasmáticos y el depósito de IgG en la pared arterial. La eliminación de LB disminuye el aumento de PA, la fibrosis y el aumento de rigidez de la pared arterial frente a la infusión de AngII ${ }^{14}$.

Finalmente, los linfocitos $\mathrm{T}$ gamma-delta (LT $\gamma \delta)$, un tipo de LT (CD4- y CD8 $4^{-}$que no requieren activación por presentación antigénica y representa $0.5 \%-10 \%$ de los LT circulantes, aumentan y se activan con infusión de AngII en ratones. El déficit de $\mathrm{LT} \gamma \delta$ protege de la disfunción endotelial arterial ${ }^{15}$.

\section{b) Papel de los linfocitos en la disminución de la capacidad excretora de sodio renal}

La disminución de la capacidad natriurética durante el desarrollo de HTA se asocia a la aparición de infiltrado por leucocitos mononucleares en el espacio túbulo-intersticial. Estos favorecen el desarrollo de estrés oxidativo, reducen la biodisponibilidad de NO renal y disminuyen el flujo sanguíneo renal medular, reduciendo la excreción urinaria de sodio $^{1-3,7}$.

La deficiencia de LTCD8 ${ }^{+}$, protege la capacidad natriurética de ratones tratados con AngII. Se ha propuesto los $\mathrm{LTCD}^{+}$renales, después de activación inmune, podrían disminuir la excreción urinaria de $\mathrm{NaCl}$ por la liberación paracrina de especies reactivas de oxígeno (ROS), activando el 
cotransportador sodio-cloruro (NCC) sensible a tiazidas, causando HTA-sal-sensible ${ }^{16}$. Adicionalmente, LTCD4 ${ }^{+}$activados durante el desarrollo de HTA contribuirían a la caída de la excreción urinaria de sodio vía citoquinas producidas por LTh1 y LTh17 (IL-6, IL-17, INF- $\gamma$ y TNF $\alpha$ ), que aumentan la reabsorción de sodio en diferentes segmentos del túbulo renal ${ }^{17}$.

\section{c) Evidencia de activación de la inmunidad adaptativa en pacientes hipertensos}

Además de la evidencia de daño inflamatorio en tejidos blanco de HTA y aumento de marcadores de inflamación en pacientes hipertensos, estudios recientes han demostrado que el aumento de marcadores inflamatorios (proteína $\mathrm{C}$ reactiva e IL-6) son predictores del desarrollo de $\mathrm{HTA}^{18}$. El estudio CRIC mostró que el aumento de TNF- $\alpha$, IL-6 y la reducción en TGFb1 (factor de crecimiento transformante b1) estaban asociados a HTA resistente en pacientes con deterioro de la función renal ${ }^{19}$. Se ha demostrado aumento de $\mathrm{LTCD}^{+}$proinflamatorios en sangre de pacientes hipertensos ${ }^{20}$. Además, se ha encontrado correlación positiva entre niveles de IL-17 circulante e hipertensión, y aumento en los niveles de LTh17 circulantes en pacientes hipertensos vs. sujetos normotensos ${ }^{11,17}$. Los niveles de IL-17 son mayores en pacientes hipertensos no controlados y en hipertensos con enfermedad de mayor duración ${ }^{17,21}$.

\section{Mecanismo de activación inmune en hipertensión arterial}

La activación de respuestas linfocitarias específicas durante el desarrollo de HTA implica la existencia de un mecanismo inmunológico. El desarrollo de una respuesta inmune adaptativa sería desencadenado por antígenos generados por daño molecular (Damage-associated molecular patterns; DAMPs): biomoléculas endógenas y/o neoantígenos producidos como resultado o asociados al aumento de la PA, que inician y/o perpetúan una respuesta inflamatoria. La respuesta específica requiere que células del sistema monocito-macrofágico (MMc) actuen como células presentadoras de antígenos (APCs), macrófagos o células dendríticas (DCs) mediante moléculas del MHC. La interación APC-LT "naive" promueve la diferenciación y proliferación de LT efectores específicos ${ }^{17}$, orquestando la respuesta inmune adaptativa.

\section{Monocito/macrófagos (MMc)}

Los monocitos en sangre periférica de pacientes con HTA están activados y presentan un fenotipo proinflamatorio comparándolos a normotensos. También ratas con HTA muestran aumento de DCs y MMc con TNF- $\alpha$ activado, en aorta y tejido renal. Ellos son activados por el endotelio vascular sometido a mayor estiramiento durante la $\mathrm{HTA}^{17}$. El déficit funcional de MMc, o su eliminación, previene parcialmente el aumento de la PA, disminuye la disfunción y atenúa la producción de ROS en la pared vascular en respuesta a AngII o MNa. Además, la transferencia adoptiva de monocitos a ratones deficientes de MMc, restablece la acción prohipertensiva de AngII. Dado que AngII y/o Aldosterona (Aldo) pueden activar los MMc, provocando la generación de ROS en el músculo liso de la pared vascular, se propone que la ausencia de MMc protege la función vascular favoreciendo mayor biodisponibilidad de NO endotelial frente a estímulos prohipertensivos ${ }^{17}$.

Los macrófagos tienen mucha plasticidad, su fenotipo es caracterizado como M1 (proinflamatorio) y M2 (inmunomodulador). La disminución de la relación M1/M2 normaliza la HTA en ratas espontáneamente hipertensas. Al contrario, aumentando M1 se desarrolla HTA inducida por An$\mathrm{gII}^{17}$. Dieta rica en sal promueve la diferenciación macrofágica hacia fenotipo M1, disminuyendo la diferenciación hacia fenotipo $\mathrm{M} 2$ reparativo y supresor de la función de LT efectores.

Células dendríticas (DCs): Derivadas de la médula ósea, presentan los antígenos a los LT. Tienen la capacidad de capturar, procesar y presentar antígenos en contexto MHCI y MHCII, para la activación y polarización linfocitaria ${ }^{17}$. Patrullan los vasos sanguíneos y órganos, mantienen la tolerancia inmunológica de los tejidos, pero en patologías como arteriosclerosis, enfermedad renal crónica o hipertensión activan LT. El bloqueo de su función activadora protege parcialmente el desarrollo de HTA inducida por AngII o Mna; transferir DCs obtenidas de un ratón hipertenso a un ratón sano aumenta la sensibilidad para desarrollar HTA por AngII. Esto propone que DAMPs y neoantígenos capturados por las DCs durante la HTA, y/o generados por las propias DCs, promovería la proliferación y polarización del $\mathrm{LT}^{17}$.

Adicionalmente, aumentos de estrés oxidativo en las DCs contribuiría a la formación de neoantígenos, como derivados de la peroxidación 
lipídica (isolevuglandinas o $\gamma$-cetoaldehidos) que modifican proteínas celulares. Antioxidantes farmacológicos (scavenger) de peroxidación lipídica reducen la PA, la disfunción endotelial, y aminora el aumento de rigidez de la pared arterial en animales de experimentación. En la misma línea, DCs de pacientes con HTA presentan peroxidación lipídica aumentada.

En HTA experimental sal-sensible, las DCs expuestas al aumento de sodio extracelular aumentan la expresión y actividad de la NADPH oxidasa, aumentando ROS, peroxidación lipídica y la formación de aductos proteína-isoketales ${ }^{17}$. Su capacidad de responder al aumento de sodio extracelular dependería de la expresión del Canal de Sodio Epitelial en la membrana celular, que mediaría la entrada de sodio y la activación de vías de señalización intracelular. Estudios experimentales muestran que la activación de DCs es necesaria para producir disfunción endotelial como respuesta a estímulos prohipertensivos ${ }^{22}$.

La activación del MR en DCs aumenta su actividad proinflamatoria, potenciando el desarrollo de autoinmunidad tipo $\mathrm{T}_{\mathrm{h}} 17$ siendo un mecanismo que contribuye al desarrollo de HTA y/o daño de tejido blanco ${ }^{23}$.

Nuestros estudios muestran que eliminando DCs en ratones genéticamente modificados, se previene el desarrollo de HTA en respuesta a AngII/Aldosterona+ $\mathrm{MNa}$, protegiendo de hipertrofia cardíaca, la disminución de la capacidad natriurética y la natriuresis por presión, como se observa en estados aumentados de AngII circulante. Esta eliminación en animales con HTA ya establecida por dos semanas, causa disminución de PA en menos de $24 \mathrm{~h}$. Esto muestra que las DCs modulan la función renal rápidamente, aunque los mecanismos de inmunidad adaptativa ya estuvieran desencadenados. Su acción implicaría efecto localizado en el tejido renal, pero incluiría acciones en el sistema nervioso central, en áreas relacionadas al control de la PA y del tono simpático $^{24,25}$. Así, eliminando células mieloides (microglia) de estas áreas, se protegerían parcialmente los aumentos de PA por AngII y en animales con HTA sal-sensible ${ }^{26}$.

\section{Cloruro de sodio ( $\mathrm{NaCl}$ )}

En la HTA sal sensible, la retención de sodio es acompañada de acumulación isotónica de agua, lo que llevaría a la expansión del medio extracelular.
Sin embargo, estudios experimentales y humanos, indican que la alta ingesta de sal acumularía sodio en el compartimiento intersticial de la piel y del tejido muscular esquelético. En ellos el sodio se asocia con proteoglicanos aumentando la osmolaridad intersticial ${ }^{27}$. Se ha propuesto que los proteoglicanos intersticiales actuarían amortiguando el balance positivo de sodio, donde los MMc participan como reguladores de su almacenamiento intersticial, mediante mecanismos que incluyen cambios locales de tonicidad extracelular y factores de transcripción intra MMc, que se activan por aumentos de la osmolaridad ${ }^{28}$. Adicionalmente ellos frente a ingesta aumentada de sal responden incrementando la expresión de ciclooxigenasa 2 y la síntesis de prostaglandina $\mathrm{E}$, la que tiene un efecto protector de la natriuresis renal, modulando la linfoangiogénesis en la piel ${ }^{29}$.

\section{Estrés oxidativo}

Además de inflamación, en hipertensos aumenta el estrés oxidativo, generando un envejecimiento vascular precoz. Muestran un estrés oxidativo crónico que se demuestra por altos niveles de malonil dialdehido y de F-8 isoprostanos, por una baja capacidad antioxidante del plasma y reducción de la actividad de enzimas antioxidantes como superóxido dismutasa, catalasa y glutatión peroxidasa en los glóbulos rojos y reservas de glutatión ${ }^{30}$. Conocemos que AngII aumenta el estrés oxidativo mediado por sistemas oxidativos $\mathrm{NADP} / \mathrm{NADPH}^{31}$.

\section{Resumiendo (Figura 1)}

Para que haya HTA deben activarse linfocitos T (LT CD8+, LTh, LT $\gamma \delta$ ) productores de citoquinas que inducen disfunción endotelial, alteración del manejo de natriuresis renal, hipertrofia de miocitos e inflamación y daño tisular. La activación se realiza por APCs en el complejo de Histocompatibilidad que son estimuladas por DAMPs, neoantígenos y estrés oxidativo. La HTA por AngII o Mineralocorticoides + sal, genera nuevos DAMPs que perpetúan el ciclo. Hay elementos protectores como LTreg, Monocitos/Macrófagos M2, IL10; que se ven disminuidos en la HTA.

\section{Terapéutica}

En HTA se ha usado 2 tipos de terapias: las No-farmacológicas y las farmacológicas. 




Figura 1. Hipertensión arterial, Sistema inmune y terapéutica. En la hipertensión arterial (HTA), se generan neoantígenos y DAMPs en tejidos diana como la vasculatura, el corazón y riñón, estos neoantígenos son captados por APCs, las cuales, interactúan con un LT naive, promoviendo su diferenciación hacia un LT efector, en donde LT 1, LT 17, LTCD8 ${ }^{+}$y LT $\gamma \delta$, perpetúan la condición de HTA modulando la RPT, natriuresis e inflamación tisular mientras que LTreg disminuyen esta condición. Por otro lado, APCs directamente pueden modular la HTA modificando la RPT y Natriuresis. En Obesidad se observa una aumento del estado inflamatorio, promoviendo la HTA, lo cual puede ser atenuado farmacológicamente y no farmacológicamente modulando las células del sistema inmune. Angll, Angiotensina II; ROS, Especies Reactivas de Oxígeno; DAMPs, Patrones Moleculares Asociado a Daño; RPT, Resistencia Periférica Total; NO, Óxido nítrico; DCs, Células Dendríticas; M1, Macrófagos tipo M1, APC: Células Presentadoras de Antígeno; LT, Linfocitos T; LTh, Linfocitos T helper; LTreg, Linfocitos T reguladores.

\section{Algunas no-farmacológicas}

Obesidad: El sobrepeso y la obesidad son estados inflamatorios. Frecuentemente la HTA está asociada con obesidad y el aumento del índice de masa corporal (BMI) que es uno de los factores de riesgo más importantes para desarrollarla ${ }^{32}$. Desde este punto de vista, en hipertensos, la meta es reducir el peso.

En obesidad hay una activación crónica en bajo grado del sistema inmunitario, con inflamación metabólica provocada por los macrófagos. La expansión del tejido adiposo en la obesidad induce una disfunción en la producción de adipokinas (Leptina, Adiponectina, Resistina, entre otras), aumentando citoquinas proinflamatorias como TNF- $\alpha$ y IL- 6 .

Además se produce un temprano aumento de macrófagos, siendo el componente inmune más abundante del tejido adiposo, llegando a ser el 12\% en la grasa visceral de obesos y un $4 \%$ en delgados. En pacientes delgados predominan los macrófagos
M2, mientras que en obesos predominan los M1, siendo reversible con adiponectina. Dietas ricas en grasas y/o balance calórico positivo, provocan aumento de DCs en tejido adiposo con infiltración y polarización $\mathrm{LT}_{\mathrm{h}} 1$ y $\mathrm{LT}_{\mathrm{h}} 17$ y disminución de LTreg, generando diferenciación de macrófagos hacia fenotipo $\mathrm{Ml}^{33}$.

Otro factor conducente a desarrollar HTA en obesos es la microbiota intestinal, cambiante según la composición de la dieta y balance calórico modulando la inmunidad local y sistémica, particularmente adiposo-visceral. Estudios muestran que trasplantar microbiota intestinal desde animales hipertensos a normotensos aumenta la PA. Aumentando la ingesta de sal se altera la composición de la microbiota, activando la inmunidad.

La microbiota actúa en parte por antígenos (lipopolisacáridos y péptidoglicanos) que entran desde el lumen intestinal a la circulación portal desencadenando una reacción inflamatoria sub-aguda endotoxémica ${ }^{34}$. En HTA experimental 
aumenta la permeabilidad intestinal, por la vía paracelular, favoreciendo el ingreso de antígenos de la microbiota intestinal generando un estado inflamatorio subagudo ${ }^{35}$. Además, los productos de fermentación bacteriana de la fibra dietaria, especialmente ácidos grasos de cadena corta (butirato, propionato, acetato), tienen efectos antiinflamatorios. Terapias con ácidos grasos de cadena corta reducen la PA de roedores, la inflamación sistémica, la disfunción endotelial, el daño cardiaco y vascular, atenuando la HTA inducida por AngII ${ }^{36}$.

\section{Ejercicio}

Estudios clínicos y experimentales demuestran que la actividad física, de intensidad mayor, disminuye la inflamación local en el músculo esquelético, mejora la capilarización, el flujo sanguíneo y la utilización del oxígeno, aumentando su capacidad funcional. Observaciones indican que la estimulación adrenérgica por catecolaminas también participaría en la respuesta antiinflamatoria que produce el ejercicio, vía receptores $\beta 2$, modulando la respuesta inflamatoria innata de los macrófagos ${ }^{37}$.

Los beneficios del ejercicio regular son atribuibles a una disminución en la inflamación crónica y la producción de mediadores inflamatorios ${ }^{38}$.

Ejercicios de flexibilidad, potencia y aeróbicos reducen los biomarcadores de ROS aumentando las enzimas antioxidantes. De igual modo se observa disminución de la PA debido a cambios en factores hemodinámicos, hormonales, neuronales, y a un aumento del NO, mejorando el sistema antioxidante y disminuyendo la inflamación. Existen pocos estudios sobre entrenamiento de flexibilidad que es especialmente útil para disminuir la PA ya que la pobre flexibilidad está asociada a rigidez $\operatorname{arterial}^{39}$.

\section{Terapia farmacológica}

AngII induce inflamación, por lo tanto terapias tipo ARAII (antagonistas del receptor de AngII tipo I $\left[\mathrm{AT}_{1} \mathrm{R}\right]$ ) y/o moduladores del SRAA la reducirían. Fármacos cardiovasculares convencionales (estatinas, bloqueadores de canales de calcio, inhibidores de la enzima convertidora de Angiotensina (IECA)/ARAII, antialdosterónicos) muestran efectos antiinflamatorios adicionales relacionados con sus propiedades hipotensoras. El bloqueo del $\mathrm{AT}_{1} \mathrm{R}$ tiene efectos antiinflama- torios al antagonizar la AngII y al favorecer el predominio del receptor $\mathrm{AT}_{2}$ aumenta la producción de NO.

De igual forma, Amlodipino tendría efectos antiinflamatorios como los IECA y ARAII, disminuyendo los niveles séricos de citoquinas proinflamatorias en pacientes hipertensos.

Estatinas regulan a la baja el AT $R$, aumentan el $\mathrm{NO}$ por regulación positiva de la NO-sintasa, inhiben la síntesis de endotelina-1 y la producción de citoquinas proinflamatorias bloqueando la vía NF$\kappa \beta$. Estudios informan reducciones de $2-5 \mathrm{mmHg}$ de PA sistólica con tratamiento y su mayor efecto dependería de altos valores previos de PA. Tienen sinergia junto con IECA o ARAII, sugiriendo que sus propiedades antiinflamatorias-antihipertensivas serían el resultado de inhibición del SRAA.

El micofenolato disminuye la PA o puede prevenir desarrollar HTA, con lo que abre una ventana terapéutica con drogas anti-citoquinas proinflamatorias o como anticuerpos monoclonales.

En la literatura observamos resultados diversos en PA con fármacos antiinflamatorios en enfermedades inmuno-mediadas (Lupus, Psoriasis y Artritis Reumatoide), pero hasta el momento, no existe un anticuerpo monoclonal aprobado para HTA, ya sea anti TNF- $\alpha$, anti IL- 1 b, 6,12 o $23^{40}$.

Estos antecedentes, sitúan a las células del sistema inmune como agentes centrales y causales en la HTA y su estado inflamatorio, promoviendo la investigación hacia una terapéutica dirigida a este nuevo mecanismo patológico.

\section{Referencias}

1. Caillon A, Schiffrin EL. Role of inflammation and immunity in hypertension: recent epidemiological, laboratory, and clinical evidence. Curr Hypertens Rep. 2016; 18: 21.

2. Crowley SD, Song Y-S, Lin EE, Griffiths R, Kim H-S, Ruiz P. Lymphocyte responses exacerbate angiotensin II-dependent hypertension. Am J Physiol Regul Integr Comp Physiol. 2010; 298: R1089-97.

3. Mattson DL, Lund H, Guo C, Rudemiller N, Geurts AM, Jacob $\mathrm{H}$. Genetic mutation of recombination activating gene 1 in Dahl salt-sensitive rats attenuates hypertension and renal damage. Am J Physiol Regul Integr Comp Physiol. 2013; 304: R 407-14.

4. Trott DW, Thabet SR, Kirabo A, Saleh MA, Itani H, 
Norlander AE, et al. Oligoclonal CD8+ T cells play a critical role in the development of hypertension. Hypertension. 2014; 64: 1108-15.

5. Ma F, Feng J, Zhang C, Li Y, Qi G, Li H, et al. The Requirement of CD8 + T Cells To Initiate and Augment Acute Cardiac Inflammatory Response to High Blood Pressure. The Journal of Immunology. 2014; 192: 336573.

6. Sun X-N, Li C, Liu Y, Du L-J, Zeng M-R, Zheng X-J, et al. T-Cell Mineralocorticoid Receptor Controls Blood Pressure by Regulating Interferon-Gamma. Circulation Research. 2017; 120: 1584-97.

7. Schiffrin EL. Immune mechanisms in hypertension and vascular injury. Clin Sci (Lond). 2014; 126 (4): 267-74.

8. Kossmann S, Schwenk M, Hausding M, Karbach SH, Schmidgen MI, Brandt M, et al. Angiotensin II-induced vascular dysfunction depends on interferon- $\gamma$-driven immune cell recruitment and mutual activation of monocytes and NK-cells. Arteriosclerosis, Thrombosis, and Vascular Biology. 2013; 33:1313-19.

9. Coles B, Fielding CA, Rose-John S, Scheller J, Jones SA, O’Donnell VB. Classic Interleukin-6 Receptor Signaling and Interleukin-6 trans-Signaling Differentially Control Angiotensin II-Dependent Hypertension, Cardiac Signal Transducer and Activator of Transcription-3 Activation, and Vascular Hypertrophy in Vivo. The American Journal of Pathology. 2010; 171: 315-25.

10. Amador CA, Barrientos V, Peña J, Herrada AA, González $M$, Valdés $S$, et al. Spironolactone decreases DOCA-salt-induced organ damage by blocking the activation of $\mathrm{T}$ helper 17 and the downregulation of regulatory T lymphocytes. Hypertension. 2014; 63: 797803.

11. Saleh MA, Norlander AE, Madhur MS. Inhibition of Interleukin-17A, But Not Interleukin-17F, Signaling Lowers Blood Pressure, and Reduces End-Organ Inflammation in Angiotensin II-Induced Hypertension. JACC: Basic to Translational Science. 2016; 1: 606-16.

12. Barhoumi T, Kasal DA, Li MW, Shbat L, Laurant P, Neves MF, Paradis P, Schiffrin EL. T Regulatory lymphocytes prevent angiotensin II-induced hypertension and vascular injury. Hypertension. 2011;57:469-76.

13. Iulita MF, Duchemin S, Vallerand D, Barhoumi T, Alvarez F, Istomine $\mathrm{R}$, Laurent $\mathrm{C}$, Youwakim J, Paradis P, Arbour N, Piccirillo CA, Schiffrin EL, Girouard H. $\mathrm{CD} 4$ + regulatory $\mathrm{T}$ lymphocytes prevent impaired cerebral blood flow in angiotensin II-induced hypertension. Journal of the American Heart Association. 2019;8:e009372.

14. Chan CT, Sobey CG, Lieu M, Ferens D, Kett MM, Diep $\mathrm{H}$, et al. Obligatory role for $\mathrm{B}$ cells in the development of angiotensin II-dependent hypertension. Hypertension 2015; 66:1023-33.

15. Caillon A, Mian MOR, Fraulob-Aquino JC, Huo K-G, Barhoumi T, Ouerd S, et al. $\gamma \delta$ T Cells Mediate Angiotensin II-Induced Hypertension and Vascular Injury. Circulation. 2017; 135: 2155-62.

16. Liu Y, Rafferty TM, Rhee SW, Webber JS, Song L, Ko B, et al. CD8+ T cells stimulate $\mathrm{Na}-\mathrm{Cl}$ co-transporter NCC in distal convoluted tubules leading to salt-sensitive hypertension. Nat Commun. 2017; 8: 14037.

17. Norlander AE, Madhur MS, Harrison DG. The immunology of hypertension. J Exp Med. 2018; 215: 21-33.

18. Jayedi A, Rahimi K, Bautista LE, Nazarzadeh M, Zargar MS, Shab-Bidar S. Inflammation markers and risk of developing hypertension: A meta-analysis of cohort studies. Heart. 2019; 105: 686-92.

19. Chen J, Bundy JD, Hamm LL, Hsu CY, Lash J, Miller ER $3^{\text {rd }}$, et al. Inflammation and apparent treatment resistant-hypertension in patients with chronic kidney disease. Hypertension. 2019; 73: 785-93.

20. Youn JC, Yu HT, Lim BJ, Koh MJ, Lee J, Chang DY et al. Immunosenescent CD8+ T cells and C-X-C chemokine receptor type 3 chemokines are increased in human hypertension. Hypertension. 2013; 62 (1): 126-33.

21. Simundic T, Jelakovic B, Dzumhur A, Turk T, Sahinovic I, Dobrosevic B, et al. Interleukin 17A and Toll-like Receptor 4 in Patients with Arterial Hypertension. Kidney and Blood Pressure Research. 2017; 42: 99-108.

22. Barbaro NR, Foss JD, Kryshtal DO, Tsyba N, Kumaresan S, Xiao L, et al. Dendritic Cell Amiloride-Sensitive Channels Mediate Sodium-Induced Inflammation and Hypertension. Cell Reports. 2017; 21: 1009-20.

23. Herrada AA, Contreras FJ, Marini NP, Amador CA, González PA, Cortés CM, et al. Aldosterone Promotes Autoimmune Damage by Enhancing Th17-Mediated Immunity. The Journal of Immunology. 2010; 184:19102 .

24. Hevia D, Araos P, Prado C, Fuentes Luppichini E, Rojas M, Alzamora R, et al. Myeloid CD11c+ Antigen-Presenting Cells Ablation Prevents Hypertension in Response to Angiotensin II Plus High-Salt Diet. Hypertension. 2018; 71: 709-18.

25. Araos P, Prado C, Lozano M, Figueroa S, Espinoza A, Berger $\mathrm{T}$ et al. Dendritic cells are crucial for cardiovascular remodeling and modulate neutrophil gelatinase-associated lipocalin expression upon mineralocorticoid receptor activation. J Hypertens. 2019; 37: 1482-92.

26. Shen XZ, Li Y, Li L, Shah KH, Bernstein KE, Lyden P, et al. Microglia participate in neurogenic regulation of hypertension. Hypertension 2015; 66: 309-16.

27. Titze J, Bauer K, Schafflhuber M, Dietsch P, Lang R, 
Schwind $\mathrm{KH}$, et al. Internal sodium balance in DOCA-salt rats: a body composition study. Am J Physiol Renal Physiol. 2005; 289: F793-802.

28. Choi SY, Lim SW, Salimi S, Yoo EJ, Lee-Kwon W, Lee $\mathrm{HH}$, et al. Tonicity-Responsive Enhancer-Binding Protein Mediates Hyperglycemia-Induced Inflammation and Vascular and Renal Injury. J Am Soc Nephrol. 2018; 29 (2): 492-504.

29. Machnik A, Dahlmann A, Kopp C, Goss J, Wagner H, van Rooijen N, et al. Mononuclear phagocyte system depletion blocks interstitial tonicity-responsive enhancer binding protein/vascular endothelial growth factor $\mathrm{C}$ expression and induces salt-sensitive hypertension in rats. Hypertension. 2010; 55 (3): 755-61.

30. Rodrigo R, Prat H, Passalacqua W, Araya J, Guichard C, Bächler JP. Relationship between oxidative stress and essential hypertension. Hypertens Res. 2007; 30 (12): 1159-67.

31. Fukai T, Siegfried MR, Ushio-Fukai M, Griendling KK, Harrison DG. Modulation of extracellular superoxide dismutase expression by angiotensin II and hypertension. Circ Res. 1999; 85: 23-8.

32. Guzik TJ, Touyz RM. Oxidative Stress, Inflammation, and Vascular Aging in Hypertension. Hypertension 2017; 70: 660-67.

33. Mraz M, Haluzik M. The role of adipose tissue immune cells in obesity and low-grade inflammation. J Endocrinol. 2014; 222 (3): 113-27.

34. Smiljanec K, Lennon SL. Sodium, Hypertension, and the Gut: Does the Gut Microbiota Go Salty? Am J Physiol Circ Physiol. 2019; 317 (6): H1173-H1182.

35. Santisteban MM, Qi Y, Zubcevic J, Kim S, Yang T, Shenoy V, et al. Hypertension-Linked Pathophysiological Alterations in the Gut. Circ Res. 2017; 120: 312-23.

36. Kim S, Kim JH, Park BO, Kwak YS. Perspectives on the therapeutic potential of short-chain fatty acid receptors. BMB Rep. 2014; 47 (3): 173-8.

37. Ortega E, Gálvez I, Martín-Cordero L. Adrenergic Regulation of Macrophage-Mediated Innate/Inflammatory Responses in Obesity and Exercise in this Condition: Role of $\beta 2$ Adrenergic Receptors. Endocr Metab Immune Disord Drug Targets. 201919 (8): 1089-99.

38. Chuong P, Wysoczynski M, Hellmann J. Do Changes in Innate Immunity Underlie the Cardiovascular Benefits of Exercise? Front Cardiovasc Med. 2019; 6: 70.

39. Korsager Larsen M, Matchkov VV. Hypertension and physical exercise: The role of oxidative stress. Medicina (Kaunas). 2016; 52 (1): 19-27.

40. Tanase DM, Gosav EM, Radu S, Ouatu A, Rezus C, Ciocoiu M, et al. Arterial Hypertension and Interleukins: Potential Therapeutic Target or Future Diagnostic Marker? Int J Hypertens. 2019; 2019: 3159283. 